\title{
Identification of Earth-directed partial halo coronal mass ejections
}

\author{
XuePu Zhao \\ W. W. Hansen Experimental Physics Laboratory, Stanford University, Stanford, CA \\ 94305-4085, USA email: xpzhao@solar.stanford.edu
}

\begin{abstract}
The very weak storm-effectiveness of frontside partial halo coronal mass ejections (FPH CMEs) suggests that many, if not most, FPH CMEs are not Earth-directed. Based on the cone model (Zhao et al., 2002) we put forward a method to identify Earth-directed partial halo CMEs from FPH CMEs.
\end{abstract}

\section{The Earthward direction of halo-type CMEs}

CMEs with apparent angular span greater than $120^{\circ}$ are termed as halo-type CMEs in what follows. The threshold of $120^{\circ}$ is selected somewhat arbitrary (see next section).

The Earthward direction of a halo-type CME is a necessary condition for the halotype CME to be storm-effective. Identification of Earth-directed halo-type CMEs is thus an important task in the research of the space weather. By "Earthward direction" or "Earth-directed" here we mean that there is a component of the halo-type CME that is able to hit the Earth, though the central axis of the CME may not be aligned with the line-of-sight.

Halo-type CMEs are interpreted as the result of the Thompson scattering of Sun's white light along the line-of-sight caused by a broad shell or bubble of dense plasma which is ejected in the frontside or backside of the Sun (Howard et al., 1982). Observations show that limb CMEs propagate radially beyond the first couple of solar radii, and that many limb CMEs maintain their angular width constant. Therefore halo-type CMEs with angular span of $360^{\circ}$ and having associated near surface activities, the frontside full halo (FFH) CMEs, are expected to be Earth-directed because the halos of FFH CMEs cover Sun's disk center, the Earth's projection in Sun's disk. The fact that all FFH CMEs observed in 1997 have associated interplanetary counterparts near the Earth and geomagnetic storms (Zhao and Webb, 2003) confirms that FFH CMEs are indeed Earthdirected. The storm-effectiveness for both FFH and FPH CMEs significantly weaker than for FFH CMEs alone (See Table 1) suggests, however, that many, if not most, FPH CMEs are not Earth-directed. It may improve the space weather forcasting if we can identify Earth-directed partial halo CMEs from all FPH CMEs.

\section{Identifying Earth-directed partial halo CMEs}

There are studies for identifying Earth-directed partial halo CMEs. Webb et al used the angular span of $140^{\circ}$ as a threshold for selecting partial halo CMEs and found that the FPH CMEs between December 1996 and June 1997 are storm-effective when the source region in the low corona is within $\sim 0.5$ radii of the Sun's disk center. Lyons et al. selected the angular span that exceeds $100^{\circ}$ and encompasses one or the other of the solar poles. Thompson et al used a similar criterion but with a higher threshold of 


\begin{tabular}{|c||c|c|c|c|}
\hline $\begin{array}{c}\text { Number of } \\
\text { CMEs }\end{array}$ & $\begin{array}{c}\text { Number of } \\
\text { Storms }\end{array}$ & $\begin{array}{c}\text { Storm- } \\
\text { effectiveness }\end{array}$ & Reference & Remarks \\
\hline \hline 132 & 59 & 0.45 & Wang et al., 2002 & FFH \& FPH, Kp > 5 \\
132 & 26 & 0.20 & Wang et al., 2002 & FFH \& FPH, Kp > 7 \\
125 & 44 & 0.35 & Yermolaev .. 2003a & FFH \& FPH, Dst < -60 \\
125 & 50 & 0.40 & Yermolaev .. 2003b & FFH \& FPH, Dst < -50 \\
70 & 45 & 0.64 & Zhao \& Webb 2003 & FFH, Dst < -50 \\
49 & 35 & 0.71 & Zhao \& Webb 2003 & Centered FFH, Dst <-50 \\
\hline
\end{tabular}

Table 1. Storm-effectiveness of frontside halo-type CMEs

$120^{\circ}$ (Webb et al., 2000 and references therein). By comparing the number of cross-pole partial halo CMEs with the total number of partial halo CMEs we find that using the cross-pole constrain may exclude only $\sim 25 \%$ of all partial halo CMEs. It is necessary to develop a new method to identify Earth-directed partial halo CMEs.

FPH CMEs with angular span greater than $180^{\circ}$ would also be Earth-directed if their angular width maintains constant. However, the angular width for many CMEs does not maintain constant. In fact there are storm-effective FPH CMEs with angular span significantly less than $180^{\circ}$.

We have developed a cone model to determine the angular width of halo-type CMEs from observed white light coronal images. If the shell of dense plasma that scatter Sun's white light and form the halo in the plan of the sky can be decribed by a conical shell (Howard et al., 1982), the angular width obtained using images observed at different times should be identical. If the shell of dense plasma can be described by a spherical shell (Howard et al., 1982), the angular width obtained for different images should be different. In other words, we may determine whether the angular width increases or decreases as the partial halo CME propagates ourwardly using the cone model.

\section{Summary}

By using the cone model and observed halo-type images we may determine whether or not the angular width of a FPH CME varies. In the case of constant angular width, the Earthward direction of the FPH CME may be determined simply depend on the observed angular span greater than $180^{\circ}$. If the obtained angular width increases as time goes on the threshold for Earth-directed partial halo CMEs should be lower than $180^{\circ}$ dependent on the variation of the angular width. The further study is in progress.

\section{References}

Howard, R. A., Michels, D. J., Sheeley Jr., N. R. \& Koomen, M. J. 1982 Astrophys. J. 263, L101-L104.

Webb D. F., Cliver, E. W., Crooker, N. U., St. Cyr, O. C., \& Thompson, B. J. 2000 J. Geophys Res. 105, 7491-7508.

Zhao, X. P., Plunkett, S. P. \& Liu, W. 2002 J. Geophys. Res. 107, doi:10,1029/2001JA009143.

Zhao, X. P. \& Webb, D. F.. 2003 J. Geophys. Res. 108, doi:10.1029/2002JA009606. 


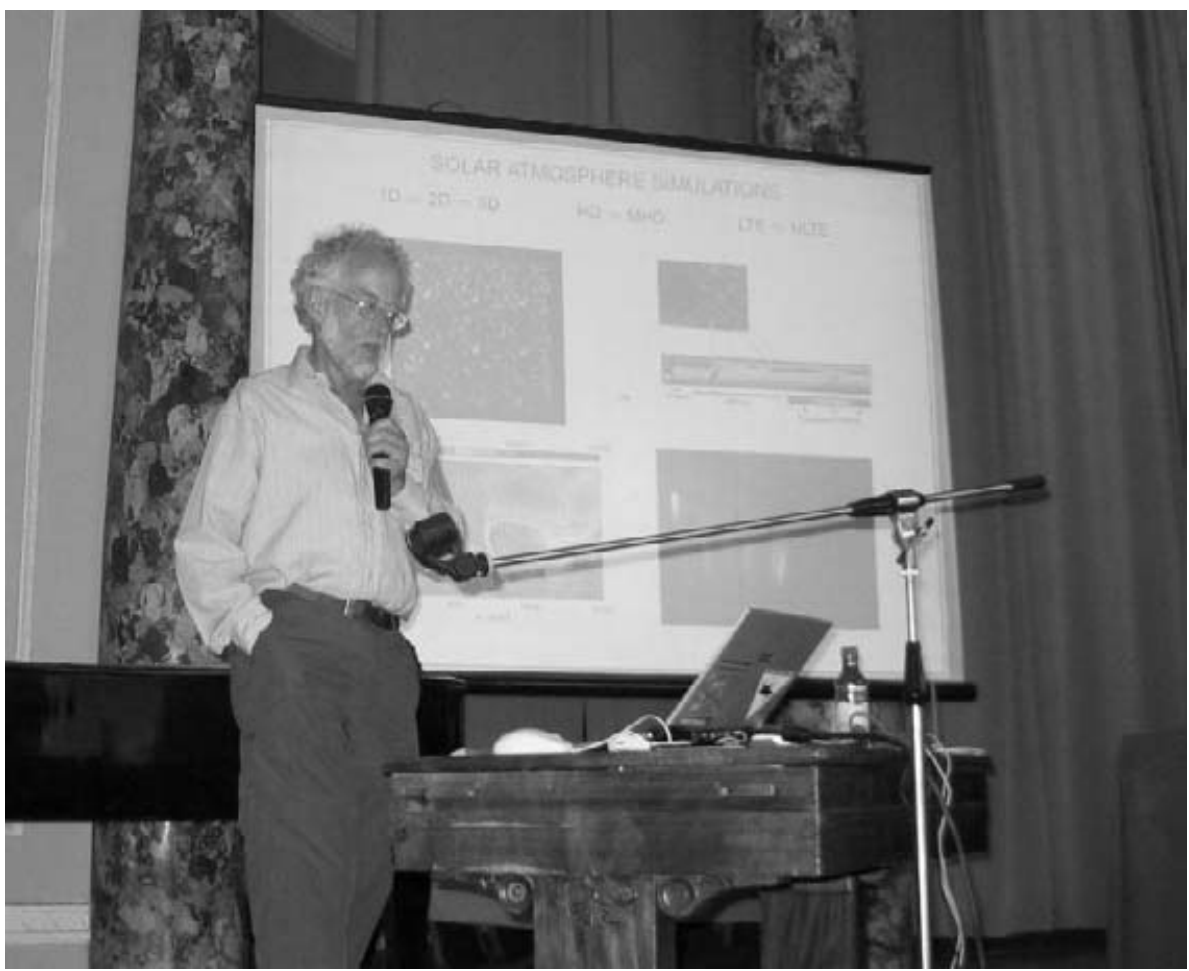

Robert Rutten (The Netherlands)

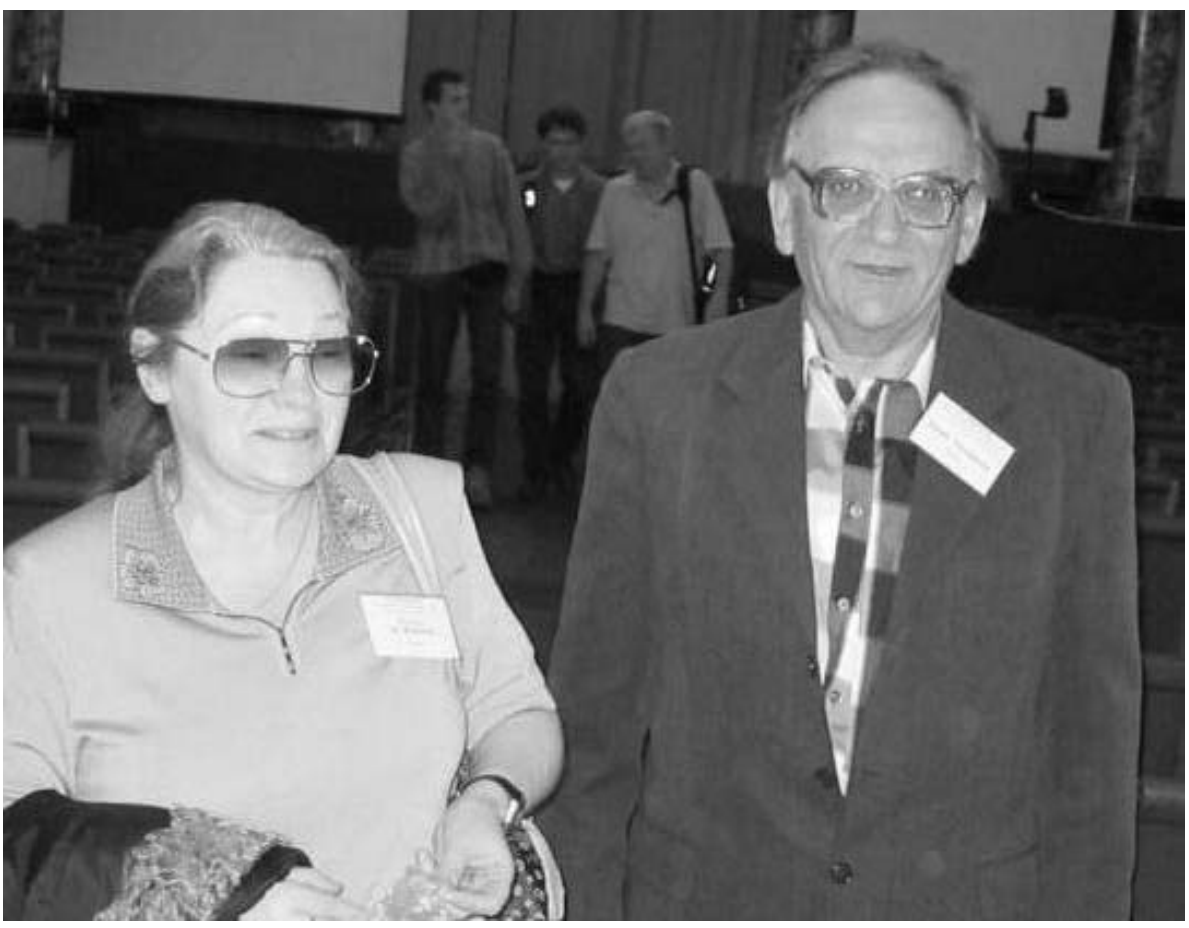

Maria Katsova (Russia) and Roald Gershberg (Ukraine) 


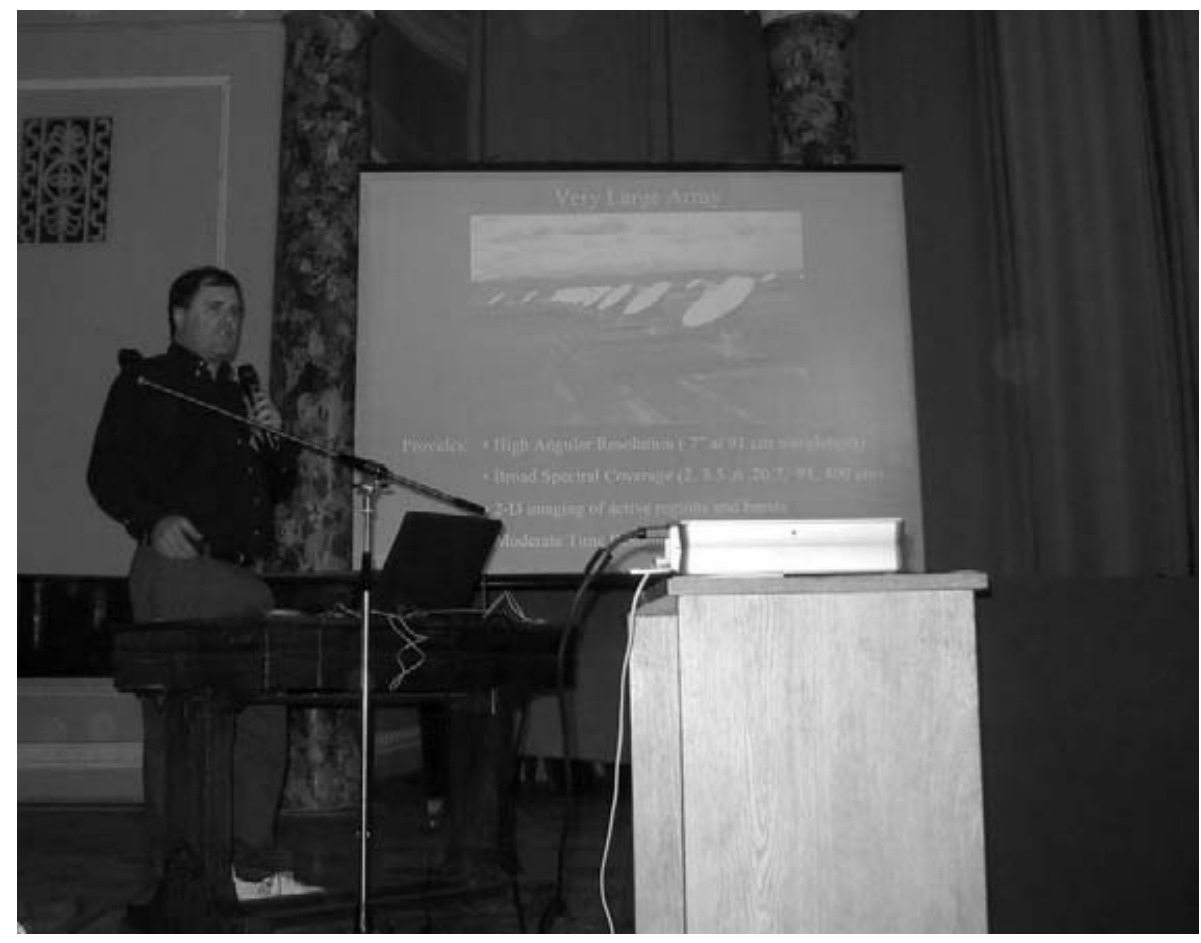

Robert Willson (USA)

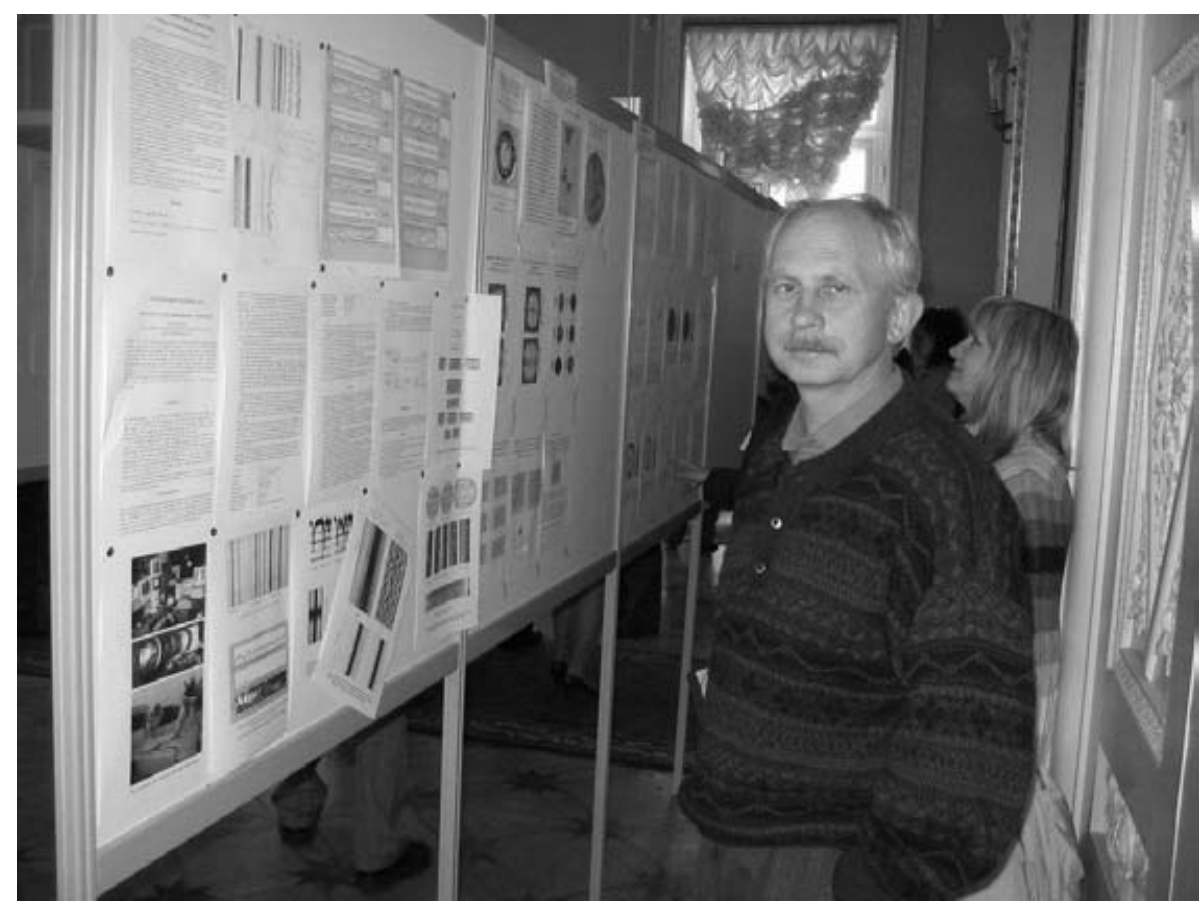

Leonid Parfinenko (Russia) 